\title{
14. Germanistik von Schulmännern: \\ Albert Bielschowskys Goethebiographie und Alfred Bieses \\ «Deutsche Literaturgeschichte»
}

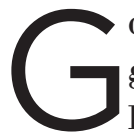

oethekult, Klassikerpflege und Biographienboom prägten auch das Programm des Verlags C.H.Beck im

Der Lorbeerkranz ist, wo er dir erscheint, ein Zeichen mehr des Leidens als des Glücks! Goethe, Torquato Tasso Kaiserreich. Oskar Beck veröffentlichte Bestseller, die dem Literaturerlebnis des deutschen Bürgertums dienten, und verbreitete wissenschaftliche Abhandlungen, die einen Kanon deutscher Literatur aus Aufklärung, Klassik und Romantik konstituierten. Er brachte, anders gewendet, das Beck'sche Verlagssignet in die eichenen Bücherschränke der bürgerlichen Bibliotheken. Höchste Detailversessenheit wurde mit nationalem Pathos, naivem Heroenkult und psychologisierenden Interpretationen verbunden. Eine geradlinige Teleologie führte die deutsche Literatur zu ihrem einsamen Höhepunkt in der Weimarer Klassik. Hier fanden die Deutschen zu sich selbst. Als Autoren holte der Nördlinger Verleger jedoch zunächst keine Repräsentanten der universitären Germanistik ins Haus, die sich gerade anschickten, die Deutsche Philologie und die Literaturwissenschaft an den Hochschulen zu etablieren. ${ }^{1}$ Wilhelm Scherer etwa, der an der Universität Straßburg das erste Germanistische Seminar gegründet hatte, veröffentlichte nicht bei C.H.Beck, sondern bei Wissenschaftsverlagen wie Weidmann in Berlin. Oskar Beck zielte mit seinen literaturgeschichtlichen und biographischen Werken nicht auf die professionalisierte Germanistik, sondern auf ein breites bürgerliches Publikum.

In den 1880er und 189oer Jahren stieg die Zahl biographischer Darstellungen zu Goethe dramatisch. Das Genie wurde mit dem Applaus der kulturprotestantischen Eliten quasi-göttlich verehrt. In Bremen predigte ein Pastor über Texte von Goethe und Schiller. ${ }^{2}$ Der Dichterfürst wurde mit der kräftigen Unterstützung jüdischer Autoren zum Symbol des neuen Reiches und zur nationalen Integrationsfigur. Die meistgelesene Goethebiographie der Epoche wurde bei C.H.Beck verlegt, und sie stammte aus der Feder von Albert Bielschowsky - einem schlesischen Oberlehrer und Spross einer jüdischen Kaufmannsfamilie, promoviert an der Universität Breslau und bis 1886 im höheren Schuldienst tätig. Als seine Schule im 
heimatlichen Brieg in jenem Jahr aufgelöst wurde, siedelte der knapp 4,0-Jährige nach Berlin über und widmete sich als Privatgelehrter seinen Goethestudien, die von einer zweibändigen Biographie gekrönt wurden. Der erste Band von «Goethe. Sein Leben und seine Werke» wurde 1895 als «Neuigkeit zu Weihnachten» veröffentlicht; der zweite sollte eigentlich rasch folgen, aber die Fertigstellung verzögerte sich, da der Autor erkrankte. Bielschowsky soll sich übernommen haben, als er auf Goethes Spuren 1894, die Apenninenhalbinsel durchwanderte. Herzbeschwerden und Schlaflosigkeit quälten ihn seitdem. Er erkrankte schwer. 1902 starb er kurz vor der Vollendung des zweiten Bandes; das nicht abgeschlossene Kapitel über den «Faust» führte der Tübinger Philosoph Theobald Ziegler zu Ende. ${ }^{3}$ Seit 1903 waren dann beide Bände auf dem Markt. 1912 erschien eine einbändige Jubiläumsausgabe. Bis 1922 waren 42 Auflagen im Umlauf, 1926 erfolgte eine postume Überarbeitung durch Walther Linden, 1930 erreichte der erste Band die 46., der zweite die 45. Auflage. Mit mehr als 14,0000 verkauften Exemplaren war Bielschowskys «Goethe» das «Hausbuch des deutschen Bildungsbürgertums mit all den vielberedeten Klischees, repräsentativ mit ziseliertem Lederrücken und Goldschnitt». ${ }^{4}$

Wie fand der Autor zu C.H.Beck? Albert Bielschowsky war dem Verleger durch die Neuauflage der Biographie und Briefedition von Lilli Schönemann verbunden, die einst als Goethes Verlobte «Lilli» Bekanntheit erlangt hatte. ${ }^{5}$ Bevor dieses Buch zu Weihnachten 1893 in den Handel kam, hatte Bielschowsky am 9. November bei Oskar Beck angefragt, ob er Interesse an einer umfassenden Goethebiographie habe. Die ersten Kapitel seien bereits geschrieben. Man wurde sich rasch einig. Beck erkannte die Chance für seinen Verlag und willigte ein. Bielschowsky versprach, «anschaulich» zu schreiben, «ohne oberflächlich zu werden». ${ }^{6}$ Sein Programm lautete, «Goethes Persönlichkeit» mit Hilfe seiner autobiographischen Schriften als «ein potenziertes Abbild der Menschheit» darzustellen. Goethe wurde zum «menschlichsten aller Menschen», zum Menschen kat' exochen. «Wer diese Ansicht teilt, wird geneigt sein, zuzugeben, dass ein Verständnis Goethes als Menschen zugleich ein tieferes Verständnis für die Menschheit überhaupt eröffnet.» ${ }^{7}$ Am 30. November 1893 hatte Bielschowsky an Oskar Beck geschrieben: «Goethes Gestalt sollte in meiner Zeichnung etwas von der göttlichen Sendung verraten, die auf ihr ruht. Kommt dies zum Gefühl oder zum Bewusstsein, dann darf man hoffen, dass Goethe dasjenige Ferment für das deutsche Geistesleben wird, das seiner weltgeschichtlichen Bedeutung entspricht. Bis jetzt ist Goethes 
Gestalt immer noch zu sehr unterirdischer Strom. Man kennt seine Existenz, bewundert sie hie und da, aber man lässt sich nicht von ihr heben und tragen.» ${ }^{8}$

\section{Albert Bielschowsky}

Diese Art der Goetheverehrung ist uns heute sehr fremd geworden. Die Helden und Heldinnen der Werke wurden «jungmädchenhaft» vergöttert und mit historischen Personen gleichgesetzt: Carlos, Clavigos Freund, ist Goethes Förderer Johann Heinrich Merck, Iphigenie Frau von Stein, Leonore Sanvitale aus «Torquato Tasso» die Herzogin Anna Amalia. Auch sonst erhebt sich bei späteren Lesern Widerspruch: «Gleich auf der ersten Seite tritt uns der Säugling Goethe als «der Gewaltige` und «der herrlichste Lichtbringer> entgegen; später liest man von einem chopinesken kleinen Wolfgang mit den funkelnden schwarzen Augen und dem klugen, bleichen Gesicht, ja sogar von einem «kleinen Schelm». Auch unterläuft Bielschowsky manches Fehlurteil, etwa in der Meinung, dass die Fremdsprachen dem Dichter nur so zugeflogen seien; Goethe war wohl der Einzige, der es fertigbrachte, «das Lateinische mit großer Fertigkeit [zu] beherrschen, ohne es eigentlich grammatisch erfasst zu haben ».»

Doch der Erfolg beschränkte sich nicht nur auf den deutschsprachigen Raum. Die zweibändige Biographie wurde ins Englische übersetzt, und in der angelsächsischen Welt fand das Werk ebenfalls begeisterte Aufnahme. Ein Rezensent in der «North American Review» bezeichnete es als die «definitive biography», die George Henry Lewes' «The Life and Works of Goethe» aus der Mitte des 19.Jahrhunderts ersetzt habe: «The portrait of the man and the poet is strikingly consistent in its gradual development within the bounds set by the contradictions of Goethe's life. The strict objectivity which makes German encyclopedias the most reliable, because the most thorough and most unbiased by personal views and

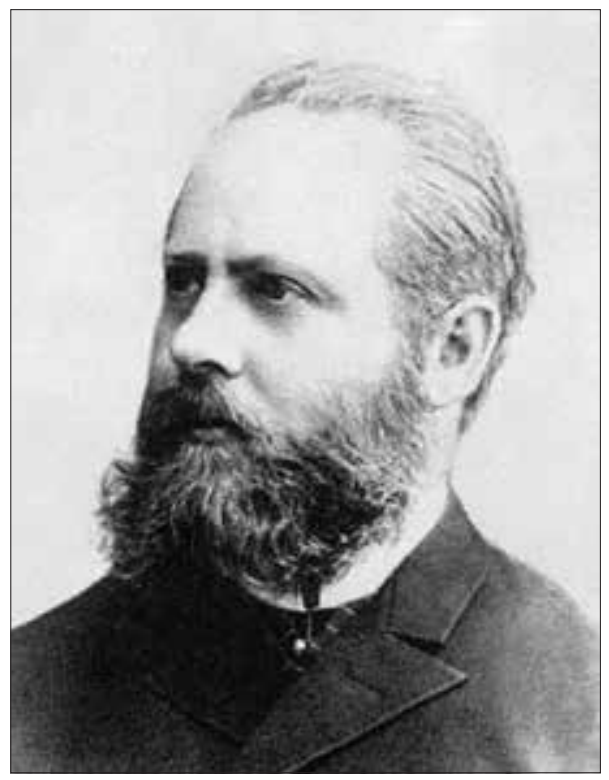

Albert Bielschowsky 
theories as distinguished from facts, is a notable feature of this work.» ${ }^{10}$

Die Rezension nennt einen der entscheidenden Gründe des Erfolgs: die Ausführlichkeit und Genauigkeit der Rekonstruktion von Leben und Werk. Die historisch-kritische Wissenschaft bemächtigte sich auf Aberhunderten von Seiten der Biographie des Dichterfürsten. Goethes Lebensgeschichte wird ebenso ausgebreitet wie die Entstehungsgeschichte seiner Werke. Im Zeitalter der Goethephilologie sollten die vielfältigen Verbindungen zwischen Leben und Werk aufgezeigt werden. «Dem gebildeten Deutschen» ging es weniger darum, "Goethe zu lesen, als über Goethe.»" Keine Aussage blieb ohne Beleg. Doch Goethe interessiert nicht nur als Gegenstand der Forschung, sondern war Teil der nationalen Identität. Ihn umgab eine sakrale Aura. ${ }^{12}$ Das Erleben der Biographie wurde zur hermeneutischen Kategorie. Folglich verlagerte sich das Rezeptionsinteresse vom Werk auf die Person. Der Autor war der Schlüssel zum Verständnis des Euvres. Bielschowskys «Hausbuch» prägte die «Goethekenntnis» und das «Goetheerlebnis» des deutschen Bürgertums, das die «Ergebnisse der gelehrten Forschung in eine effektvoll arrangierte, einfühlsame Nachdichtung des Goethe'schen Lebens» integrierte und «so die Bedürfnisse des Bildungsbürgertums nach Belehrung und romanhafter Unterhaltung gleichermaßen» befriedigte. ${ }^{13}$ Wertvoll waren auch die zuverlässigen Werkparaphrasen, die nicht nur unter Gymnasiasten dankbare Abnehmer fanden.

Bielschowskys Goethebiographie bezeugt auch die Begeisterung deutscher Juden für den Dichterfürsten. «Die Überzeugung von Goethe als dem Repräsentanten der Humanität» stand hinter dieser Goetheverehrung, «vor allem aber das Insistieren auf Goethe als einem Garanten für den humanen Kern des Deutschtums». ${ }^{14}$ So ließ noch im Jahre 1932, an Goethes 100. Todestag, der Kölner Gemeinderabbiner Dr. Kober in einer Sabbat-Predigt verlauten: «Das Judentum huldigt dem Manne, dessen höchster Ruhmestitel es war: ‘Denn ich bin ein Mensch gewesen〉, dem Dichter, der in seinem Faust jenen sittlichen Idealismus uns erschließt, der da lehrt, festen Fuß zu fassen auf realem Boden der Welt, das optimistische Glaubensbekenntnis von dem endlichen Siege des Reiches Gottes auf Erden».» ${ }^{15}$ So nimmt es nicht wunder, dass Bielschowskys «Goethe» nicht nur ein beliebtes Geschenk zur Konfirmation war, sondern auch zur Bar- und Bat-Mizwa-Feier. ${ }^{16}$ Das Bekenntnis zu dem Dichterfürsten integrierte die deutschen Juden in das neue Reich: «Ohne Goethe kein Bismarck! ohne Goethe kein Deutsches Reich! Dass die Deutschen politisch ein Volk werden konnten, dazu mussten sie erst geistig ein Volk sein und 
als ein Volk sich fühlen - gemeinsam in Sprache, gemeinsam in Bildung und - so möchten wir gerne hinzufügen dürfen: gemeinsam auch im Glauben.» Bielschowsky fuhr fort: «So ist Goethes «reine Menschlichkeit» schließlich doch das Ziel, dem wir zustreben müssen. In diesem Sinne war er der erste Statthalter im Reich des deutschen Geistes, der Reichskanzler im Geiste des alten Deutschland, wie Weimar durch ihn unsere erste geistige Reichshauptstadt geworden ist.» ${ }^{17}$ Bielschowsky artikulierte in dieser Biographie das Selbstverständnis eines gebildeten jüdischen Bürgertums, das sich zu dem wilhelminischen Obrigkeitsstaat bekannte.

Oskar Beck interessierte die Frage nicht, ob seine Autoren Juden waren oder nicht. Aber der Aufstieg der Antisemiten hatte sehr wohl zur Folge, dass seine jüdischen Autoren Anwürfen und Angriffen ausgesetzt waren. So bemerkte Richard M. Meyer, dass es keinen Grund gebe, die öffentliche Aufnahme seiner Nietzschebiographie zu beklagen, aber es verletze ihn, «dass konservative, agrarische, antisemitische Blätter lediglich aus politischer Voreingenommenheit» ihn persönlich beschimpfen ließen, «ohne dass ein einziger Leser einmal gegen diese Unanständigkeit Einspruch» erhöbe. ${ }^{18}$

\section{Biographien, Editionen, Kommentare}

Goethe wurde nicht historisiert, sondern heroisiert. Aber Goethe stand nicht allein. «Die günstige Aufnahme, die schon der erste Band der Goethebiographie fand, legte den Gedanken nahe, ihr auch eine Schillerbiographie an die Seite zu stellen.» Karl Berger, Gymnasiallehrer aus Worms, ließ den Dichter «aus den Tiefen deutscher Volkskraft» seine schöpferische Kraft gewinnen. Als der zweite Band 1908 erschien, lag der erste, 1905 veröffentlicht, schon in der vierten Auflage vor. Rasch folgten weitere Auflagen. ${ }^{19}$ Die Biographien mit ihren ausführlichen Darstellungen von Leben und Werk, Einflüssen und Wirkungen, fanden positive Aufnahme. C.H.Beck brachte vor dem Ersten Weltkrieg in regelmäßiger Folge Lebensbeschreibungen zu den Autoren der deutschen und europäischen Höhenkammliteratur heraus - zu Herder, Grillparzer und Kleist, zu Shakespeare, Molière und Ibsen. Anderes wurde vorbereitet wie die Biographie des Romantikers Eichendorff, die Hans Brandenburg, Dozent an der Münchner Volkshochschule, zu schreiben 1910 in Aussicht stellte, tatsächlich wurde sie erst 1922 veröffentlicht. Allerdings bremste Oskar Beck die Begeisterung des Autors, der im Gegensatz zu dem Verleger die 
Marktgängigkeit seines Werkes nicht im Auge hatte. Eine solche Vita sei «weder eine Goethe- noch eine Hebbel-Biographie». Man müsse sich hüten, «die Auflage zu hoch zu machen, damit man die Chance nicht aus der Hand gibt, eine zweite Auflage zu erleben. Andererseits darf die Auflage nicht zu klein sein, da sonst der Preis zu hoch werden müsste», klärte Oskar Beck auf. Er schlug eine Auflage von 1600 Exemplaren vor. ${ }^{20}$ Der Verleger setzte nicht nur auf die auflagenstarken Titel, die gute oder sehr gute Gewinne abwarfen, sondern bediente auch speziellere Interessen, um auf dem Gebiet der deutschen Literatur gut aufgestellt zu sein. Diversifizierung der Titel und «Quersubventionierung〉 des Programms waren auch um 1900 zukunftsfähige Prinzipien der Verlagspolitik.

$\mathrm{Zu}$ den «Dichtern» traten die «Denker»: Der Berliner Philosoph Moritz Kronenberg widmete sich Kant (1897; 3. Auflage 1905, 6. Auflage 1922) und der Berliner Germanist Richard M. Meyer wandte sich Nietzsche zu (1913). ${ }^{21}$ Dass sich Kontakte zu einzelnen Autoren über die Handbücher ergaben, zeigt das Beispiel Meyers. Der junge Germanist verfasste für das «Handbuch des deutschen Unterrichts an höheren Schulen» den Band über «Deutsche Stilistik», der 1906 in erster und 1913 in zweiter Auflage erschien.

Die Werke sind heute in aller Regel vergessen, fanden aber, wie die Auflagenzahlen zeigen, unter den Zeitgenossen zahlreiche Leser, weil sie den Anspruch erhoben, die Leistungen der modernen Fachwissenschaften zu vermitteln, und weil sie die Schriftsteller und Philosophen im geistigen Milieu des Wilhelminismus verankerten. Die Werke stammten nicht von Ordinarien, sondern von fleißigen Privatdozenten, aufstrebenden Extraordinarien und gebildeten Gymnasiallehrern.

$\mathrm{Zu}$ den Biographien traten Editionen und Kommentare. Im 150. Jubiläumsjahr des Verlags erschien der zweibändige Kommentar zum «Faust» von Ernst Traumann, der in mehreren Auflagen neu bearbeitet wurde. Der Germanist Friedrich Panzer, der damals noch an der neu gegründeten Akademie für Sozial- und Handelswissenschaften, der späteren Johann Wolfgang Goethe-Universität in Frankfurt, lehrte, brachte 1913 einen Neudruck der Märchensammlung der Brüder Grimm heraus. Der Gelehrte hatte darüber hinaus einen Vertrag für das «Handbuch des deutschen Unterrichts an höheren Schulen» abgeschlossen, das von Adolf Matthias herausgegeben wurde. In dieser Reihe sollte er für den fünften Band die «Deutschen Heldensagen» bearbeiten. Panzer geriet mit beiden Projekten - der Überarbeitung der Grimm'schen Sagen und seinem Beitrag für das Handbuch - in Verzug. Es kam zu einem intensiven brieflichen Austausch zwischen Autor und Verleger, der Ende 1912 in einer heftigen Ver- 
stimmung gipfelte. ${ }^{22}$ Die Korrespondenz zeigt sinnfällig, wie umkämpft der Markt war. Bereits damals setzte man auf Jubiläen: Die Ausgabe der Märchen sollte ursprünglich zum 100-jährigen Jubiläum der Erstausgabe von 1812 veröffentlicht werden. Diederichs in Jena hatte 1912 eine Edition in zwei Bänden vorgelegt, und zwei Jahre zuvor war bei Georg Müller in München eine weitere Ausgabe veröffentlicht worden. ${ }^{23}$ Der geplante Termin war aufgrund von Panzers Säumigkeit nicht mehr zu halten, und Oskar Beck, der genau kalkulierte, war missmutig.

Am 2o. Dezember 1912 schrieb er an den «hochgeehrten Herrn Professor», es sei ihm nun gelungen, tadellose Exemplare der Titelstiche zu den beiden geplanten Bänden der Kinder- und Hausmärchen in Königsberg aufzutreiben. Er könne Panzer bald einen Probedruck übersenden und hoffe, dass dieser das Buch nun zügig fertigstellen werde. «Dass dies nicht schon zu Weihnachten möglich war, muss ich wohl am meisten bedauern, denn ich weiß wirklich nicht, wie ihm post festum und nachdem schon mehrere neue Ausgaben der Kinder- und Hausmärchen [...] auf dem Markte sind, ein auch nur einigermaßen hinreichender Absatz noch zu verschaffen ist. Es wäre mir sehr erwünscht, wenn ich nun bald Ihre «Einleitung` erhalten könnte, aus der ja wohl auch mit Deutlichkeit Ihre Begründung der eigentümlichen Bedeutung der Ausgabe, namentlich auch im Verhältnis zu den sonstigen Editionen, zu ersehen sein wird. Es wird sich ja wohl als notwendig erweisen, Publikum und Kritik hierüber eindringlich zu orientieren. Natürlich kann auch die Anzeige an den Buchhandel erst erfolgen, wenn Ihre Einleitung bzw. Ihr Vorwort vorliegt. Vielleicht wäre es möglich gewesen, wenn wir zunächst den I. Band mit aller Kraft gefördert hätten, diesen einstweilen allein auszugeben, und zwar noch zu Weihnachten. Dafür wäre aber doch vor allem die Einleitung nötig gewesen.» Oskar Beck hatte ebenso naheliegende wie klare Vorstellungen von dem Aufbau der Bände: Die Einleitung «dem II. Band am Schluss anzuhängen, hielte ich für sehr unvorteilhaft. Meines Erachtens muss die Einleitung vorn stehen, schon damit sie jedem in die Augen fällt, der das Buch in die Hand nimmt, und er dadurch sofort über dessen Absichten unterrichtet wird.» Anschuldigungen des Verfassers, der Verlag sei für die Verspätung verantwortlich, wies er unmissverständlich zurück.

Der Brief wirft ein helles Licht auf die Mühen des Verlegerberufs: «Aus einigen Andeutungen Ihrer Briefe klingt etwas wie ein Vorwurf heraus, als hätten Druckerei und Verlag die Schuld an der verspäteten Fertigstellung. Demgegenüber gestatte ich mir zu bemerken, dass doch auch die Verzögerungen Ihrer Manuskriptlieferung und der große Umfang Ihrer Korrek- 
turen sowie die langsame Erledigung der Korrekturbogen Ihrerseits aufgehalten haben. Allerdings gebe ich zu, dass die Druckerei von der gewählten Schrift, zumal dem kleineren, für die Anmerkungen verwendeten Grade, kein allzu großes Quantum zur Verfügung stellen konnte, zumal diese Schrift auch noch anderwärts Verwendung finden musste. So musste sie immer wieder auf druckfertige Bogen warten, und durch deren Reindruck wieder Schrift für den Weitersatz zu bekommen. Bei rascherer Erledigung der Korrekturen und rechtzeitiger Lieferung des Manuskripts wäre es immerhin wohl möglich gewesen, rechtzeitig fertig zu werden.»

Seinen Brief schloss Oskar Beck, indem er selbstbewusst und bestimmt seine Erwartungen an den Autor formulierte. Scheu vor dem deutschen Professor hatte er nicht. «Die Lage der Sache ist nun, wie bemerkt, etwas misslich geworden. Vielleicht wissen Sie Mittel und Wege, wie die Situation noch einigermaßen zu retten ist.» Schließlich: «Nun aber darf ich wohl sicher hoffen, dass Sie keine andere Unternehmung mehr dazwischenschieben, sondern Ihre Arbeit auf das Handbuch konzentrieren. Es würde mich nicht wenig freuen, von Ihnen bald eine gute Nachricht über die Arbeit für die Heldensagen zu erhalten.» ${ }^{24}$

Der solcherart getadelte Germanist antwortete noch am 23. Dezember. Er war verärgert und redete Oskar Beck höchst distanziert mit «hochgeehrter Herr Kommerzienrat» an. «Ihr gefälliges Schreiben vom 2o. Dezember habe ich vorgestern erhalten. Es überschüttet mich so ziemlich in Rücksicht aller Unternehmungen, zu denen ich mit Ihnen verbunden war, mit einer Flut von Vorwürfen, auf die ich so manches zu erwiedern [sic], an denen ich so manches zu berücksichtigen hätte. Ich unterlasse aber einzuwenden, was zwecklos erscheinen muss, nachdem wir einmal auf diesen Ton gekommen sind, und beschränke mich also darauf, auf das geschäftlich noch Notwendige zu bemerken.» Er schloss scharf: « Eine Verpflichtung, nicht zu veröffentlichen, bis das Handbuch fertig ist, kann ich nicht eingehen.» ${ }^{25}$ Das Handbuch wurde nie fertig. Die «Kinder und Hausmärchen in ihrer Urgestalt» erschienen 1913. Ein Jahr post festum. Die Sammlung wurde kein Erfolg. Es blieb bei einer Auflage.

\section{Alfred Biese}

Ein außerordentlicher Erfolg hingegen, durchaus mit dem der Bielschowsky'schen Goethebiographie vergleichbar, war die «Deutsche Literaturgeschichte», die der Gymnasialdirektor Alfred Biese zwischen 1907 
und 1911 in drei Bänden verfasste und die 1931 in der Überarbeitung durch Johannes Alt in der 25. Auflage erschien. Mehr als hunderttausend Exemplare des Gesamtwerks wurden verkauft. ${ }^{26}$ «Ausgezeichnet durch lebendige und schwungvolle Darstellung», wurde das Werk «zum Lieblingsbuch der Gebildeten» und zur populärsten Literaturgeschichte für ein Vierteljahrhundert. ${ }^{27}$ Biese hatte Klassische Philologie und Germanistik in Bonn, Greifswald und Kiel studiert, wo er 1878 mit einer Arbeit über Plautus und Terenz promoviert worden war. Im Anschluss unterrichtete er als Gymnasiallehrer in Kiel, Schleswig und Koblenz. 1899 wurde er Gymnasialdirektor in Neuwied und 1913 in Frankfurt am Main. Oskar Beck war schon 1897 mit der Idee an Biese herangetreten, eine zweibändige Literaturgeschichte zu schreiben, die Bielschowskys «Goethe» zur Seite gestellt werden und den gesamten Stoff der deutschen Literatur «für weite Kreise anziehend und verständlich darstellen und in die Gesamtentwicklung des deutschen Geisteslebens einordnen sollte». ${ }^{28}$ Zunächst zögerte der Gymnasiallehrer, ließ sich dann aber, als Oskar Beck ihn im Februar des Folgejahrs in Koblenz aufsuchte, umstimmen. Man beschloss, ein Konkurrenzwerk zu der von Friedrich Koch und Max Koch verfassten «Geschichte der deutschen Literatur», die 1897 im Bibliographischen Institut in Leipzig verlegt worden war, herauszubringen. Biese hatte zwar Bedenken, «war aber doch auch wiederum froh gestimmt über den ehrenvollen Antrag». Der Karneval, bei dem es im Koblenzer Casino unter 800 Masken hoch herging, fand ihn «in frohester Laune». ${ }^{29}$

Die Entstehung des Werkes verlief jedoch nicht reibungslos, oder - wie Biese selbst in seinen Erinnerungen bekannte - der Verlagsvertrag, den er «wahrlich nicht leichten Herzens» unterschrieben hatte, brachte «Kummer genug». ${ }^{30}$ Ostern 1905 fuhr der Gymnasialdirektor nach München. Die Begegnung mit Oskar Beck, der auf ihn schon früher «den Eindruck eines kenntnisreichen, klugen Mannes» gemacht hatte, ${ }^{31}$ war höchst eindrücklich: «Ich sah Beck wieder, ich war erschrocken über die schonungslose Art, wie er Druckbogen eines berühmten Autors mit Streichungen und Änderungen versah und wie er auf die kritischen Einwände, die er bei einem mir vorgelegten Bogen machte, sofort einging: Soll ich es nicht zurückschicken?», fragte er.» ${ }^{32}$ Auch das Manuskript, das Biese einreichte, entsprach nicht den Erwartungen, die der Verleger an das Werk hatte. Oskar Beck verlangte eine ausführlichere Darstellung und korrigierte den Umfang nach oben. Aus den geplanten zwei Bänden wurden drei, weil der Verleger «sehr an historischen Tatsachen hing». Als junger Redaktionsassistent ging Will Vesper über das Manuskript, der später auch als Autor 




Alfred Biese

C.H.Beck treu blieb und einige Gedichtbände veröffentlichte. Biese musste «immer wieder der Tyrannei» nachgeben und Satz um Satz nachtragen. Allerdings gestand er im Nachhinein durchaus selbstkritisch ein, dass der Erfolg dem Verleger Recht gegeben habe. «1927 waren 300000 [Einzel-] Bände ausgegeben. Das Werk hat somit seine hohe Aufgabe erfüllt, es ist dem deutschen Hause in schweren Zeiten ein Ratgeber und Wegweiser geworden.» ${ }^{33}$

Dass Oskar Beck mit dem Plan, Alfred Biese eine ausführliche Literaturgeschichte schreiben zu lassen, richtig lag, bestätigte der Verleger Freytag aus Leipzig, der den Gymnasialdirektor besuchte und sich von dem Projekt erzählen ließ. Freytag reagierte sofort und brachte 1906 eine Literaturgeschichte von Eduard Engel «mit Bomben-Reklame» heraus, die er «mit einer wohlwollenden Rezension von Adolf Matthias fast jedem Schulleiter ins Haus» schickte. ${ }^{34}$ Die Konkurrenz konnte jedoch abgewehrt werden. Bieses Geschichte - in Oskar Becks Überarbeitung - setzte sich durch. Noch 1931 bescheinigte ihr ein Rezensent ubiquitäre Verbreitung: «In vier von fünf, wenn nicht neun von zehn Fällen erfolgt auf die Frage: ‘Wo haben Sie dies oder das nachgelesen?> die Antwort: ‘Im Biese`.» ${ }^{55}$ Zwei Jahre zuvor hatte Arthur Schnitzler eine seiner «allerletzten» Portraitaufnahmen C.H.Beck zur Veröffentlichung in der Literaturgeschichte von Biese zur Verfügung gestellt. ${ }^{36}$

Die Literaturgeschichte vermittelte, wie die Dichterbiographien und die Handbücher, einem breiten Publikum Ergebnisse der neueren Forschung. Hinzu trat ein nationalpädagogisches Anliegen, die deutsche Literatur als identitätsstiftende Größe im kollektiven Bewusstsein des Bürgertums zu verankern. Literaturgeschichte wurde in patriotischer Absicht betrieben. Über Wolfram von Eschenbachs angebliche deutsche Übertragung des «Parzival» aus dem Altfranzösischen schrieb Biese, der deutsche Dichter habe «einem ungefügen Körper eine reiche Seele gegeben» und «aus einem Dümmlingsmärchen» «ein psychologisches Lebensgemälde von ernstestem Gehalt emporwachsen lassen». ${ }^{37}$

Der Zweifel an einer historistischen Literaturgeschichtsschreibung, die 
sich auf Fakten konzentrierte und unendlich viele Informationen anhäufte, erschütterte zwar die Fakultäten um 1900, nicht aber das Programm des Verlags C.H.Beck, das auf diesem Gebiet nicht die Frage nach der Bedeutung der Wissenschaft für das Leben aufgriff. ${ }^{38}$ Das Ende der liberalen Ära und die Zweifel an dem wissenschaftlichen Fortschritt wurden nur marginal reflektiert. Außenseiter hatten im literaturgeschichtlichen Programm von C.H.Beck keine Chance, vielmehr war wilhelminischer Mainstream gefragt. Verhaltene, gar zeitkritische Töne sollten die Verehrung des großen «Olympiers» ${ }^{39}$ nicht stören. Die avantgardistische und kulturkritische Goetherezeption, für die zu Beginn des neuen Jahrhunderts Georg Simmel, Houston Stewart Chamberlain und Friedrich Gundolf standen, fand nicht im Verlag C.H.Beck statt. Dort wurden «terminologisierte Systematik», «positivistische Exaktheit» und ein «überkommener Wissenschaftsstil» fortgeschrieben. ${ }^{40}$ Oskar Beck sah keinen Grund, sein Programm zu ändern. Der Erfolg gab ihm zunächst Recht. Aber das Goethebild der nachwilhelminischen Epoche prägte der Verlag nicht mehr mit. 
https://doi.org/10.17104/9783406654015-243, am 26.04.2023, 17:29:28

Open Access - (cc)EY - http://www.beck-elibrary.de/agb 